\title{
The Effect of Subfilter-Scale Physics on Regularization Models
}

\author{
Jonathan Pietarila Graham • Darryl D. Holm • \\ Pablo Mininni • Annick Pouquet
}

Received: 27 February 2010 / Revised: 28 September 2010 / Accepted: 15 October 2010 /

Published online: 4 November 2010

(C) Springer Science+Business Media, LLC 2010

\begin{abstract}
The subfilter-scale (SFS) physics of regularization models are investigated to understand the regularizations' performance as SFS models. Suppression of spectrally local SFS interactions and conservation of small-scale circulation in the Lagrangian-averaged Navier-Stokes $\alpha$-model (LANS- $\alpha$ ) is found to lead to the formation of rigid bodies. These contaminate the superfilter-scale energy spectrum with a scaling that approaches $k^{+1}$ as the SFS spectra is resolved. The Clark- $\alpha$ and Leray- $\alpha$ models, truncations of LANS- $\alpha$, do not conserve small-scale circulation and do not develop rigid bodies. LANS- $\alpha$, however, is closest to Navier-Stokes in intermittency properties. All three models are found to be stable at high Reynolds number. Differences between $L^{2}$ and $H^{1}$ norm models are clarified. For magnetohydrodynamics (MHD), the presence of the Lorentz force as a source (or sink) for circulation and as a facilitator of both spectrally nonlocal large to small scale interactions as well as local SFS interactions prevents the formation of rigid bodies in Lagrangianaveraged MHD (LAMHD- $\alpha$ ). LAMHD- $\alpha$ performs well as a predictor of superfilter-scale energy spectra and of intermittent current sheets at high Reynolds numbers. It may prove generally applicable as a MHD-LES.
\end{abstract}

The National Center for Atmospheric Research is sponsored by the National Science Foundation.

J. Pietarila Graham ( $ه)$

Max-Planck-Institut für Sonnensystemforschung, Katlenburg-Lindau, Germany

e-mail: jpietarilagraham@mailaps.org

J. Pietarila Graham

Department of Applied Mathematics \& Statistics, The Johns Hopkins University, Baltimore, MD, USA

D.D. Holm

Department of Mathematics, Imperial College London, London, UK

P. Mininni - A. Pouquet

National Center for Atmospheric Research, Boulder, CO, USA

P. Mininni

Departamento de Física, Facultad de Ciencias Exactas y Naturales, Universidad de Buenos Aires,

Ciudad Universitaria, Buenos Aires, Argentina 
Keywords LES $\cdot$ Subgrid-scale processes $\cdot$ Alpha models $\cdot$ MHD $\cdot$ Intermittency

\section{Introduction}

Computing solutions to the Navier-Stokes equations at realistic values of the Reynolds number $\left(R e \equiv U L_{0} / v\right.$, with characteristic velocity, $U$, and length-scale, $L_{0}$ and viscosity, $v$ ) for most geophysical, astrophysical, and many engineering applications is technologically infeasible. This is because the range of dynamically important length (and time) scales is quite large: from the large scale, $L_{0}$, defined by the problem down to the scales of viscous dissipation, $l_{v} \sim L_{0} R e^{-3 / 4}$. One approach is to simply cut off the smallest scales to arrive at a problem small enough for modern computational limits. A low-bandpass filter, $L: f \rightarrow \bar{f}$, replaces the velocity, $\mathbf{v}$, and pressure, $P$, with smoother, resolvable fields, $\overline{\mathbf{v}}, \bar{P}$. Their time evolution is governed by the filtered Navier-Stokes equations,

$$
\begin{aligned}
\partial_{t} \overline{\mathbf{v}}+\overline{\boldsymbol{\omega}} \times \overline{\mathbf{v}} & =-\nabla \bar{P}+v \nabla^{2} \overline{\mathbf{v}}-\nabla \cdot \bar{\tau}, \\
\nabla \cdot \mathbf{v} & =0,
\end{aligned}
$$

where $\omega=\nabla \times \mathbf{v}$ is the vorticity and $\bar{\tau}$ is the Reynolds subfilter-scale (SFS) stress tensor,

$$
\bar{\tau}_{i j}=\overline{v_{i} v_{j}}-\bar{v}_{i} \bar{v}_{j}
$$

The modeling of the unresolved stress, (2), is the main challenge of conducting such large eddy simulations (LES, see [38] for a recent review).

Regularization modeling (of the SFS stress tensor) for Navier-Stokes [8, 10, 14, 17, 18, 25, 29, 36, 50], magnetohydrodynamics (MHD) [24], Boussinesq convection [53], and inviscid cases [33] promises several advantages. For Navier-Stokes, only weak, possibly non-unique solutions have been rigorously proven to exist, and this can impact the possibility of achieving a direct numerical solution (DNS), e.g., with Fourier methods [22]. This is because to prove, even in the linear case, the convergence of a numerical solver for a partial differential equation (PDE), one assumes the PDE is well-posed (i.e., the existence of unique solutions that depend continuously on the data) [34]. A regularization has strong, unique solutions (it is well-posed) and the concerns of [22] do not prevent us from achieving a DNS of the model equations, even with Fourier methods. It is worth noting that numerical convergence of the DNS of the regularization model equations implies a grid-independent model of the Navier-Stokes equations. Additionally, the dissipative term will be unmodified (unlike many LES); the Reynolds number remains well defined (e.g., $\omega \times \overline{\mathbf{v}} / v \nabla^{2} \mathbf{v} \sim U L_{0} / v \equiv R e$ ). This is opposed to the usual approach of modeling the behavior of the flow in the limit of very large $R e$. Thus, the models may be more applicable to intermittent phenomena where the length of the inertial range can be important [46]. Since the models do not introduce the effect of the small scales in an ad hoc fashion but rather preserve the mathematical properties of the underlying equations, their application can further our understanding of turbulence and turbulence modeling. The methods are also more easily generalized to other problems (e.g., coupling to a magnetic field).

In this paper we address two separate questions. One is the question of the practical applicability of regularization models as SFS models. When addressing this question, the filter width, $\alpha$, will be placed in the inertial range and the grid spacing, $\Delta$, will be just small enough to achieve a DNS of the regularization. Our aim is then to determine how well the model's DNS reproduces a "DNS" of Navier-Stokes compared at scales larger than $\alpha$. Our second question is "How do the models work?" To answer this question, we take an 
approach that is the antithesis of a practical LES: we choose the filter width, $\alpha$, to be a large fraction of the computation domain (and, thus, a large multiple of $\Delta$ ). Such a calculation is not a LES - there is no superfilter-scale inertial range to compare with Navier-Stokes. Instead, we make a new type of study to understand the altered SFS physics introduced by the regularization. The differences in physics between the SFS model and Navier-Stokes (or MHD) is what allows the model to reduce computational cost when it is employed as a SFS model. Understanding of how the models work (or fail) can guide the development of new models as we will show.

\section{Navier-Stokes}

\subsection{LANS- $\alpha$ and Rigid Body Formation}

The first model we consider is the Lagrangian-averaged Navier-Stokes (LANS) $\alpha$-model $[10,25]$. It is derived by Lagrangian averaging fluid motions followed by application of Taylor's frozen-in turbulence approximation as the model's one and only closure: fluctuations about the Lagrangian mean smaller than $\alpha$ are swept along by the large-scale flow and are not allowed to interact with one another [24]. The model is attractive as it retains the Hamiltonian structure of Navier-Stokes, preserves Kelvin's theorem (conserves smallscale circulation in the absence of dissipation), and conserves both total energy and helicity (the correlation between the velocity, $\mathbf{v}$, and its curl, the vorticity $\omega=\nabla \times \mathbf{v}$ ) [25]. These properties are conserved in the $H_{\alpha}^{1}$ norm instead of the usual $L^{2}$ norm. This is essential when interpreting results of the model as, for example, quantities involving the square velocity, $|\mathbf{v}|^{2}$, must now be replaced with the dot product $\mathbf{v} \cdot \overline{\mathbf{v}}$ where $\overline{\mathbf{v}}$ is the filtered velocity. Physically, due to the frozen-in approximation, the model retains spectrally nonlocal interactions (important at finite $\operatorname{Re}[2,39]$ ) between the superfilter and subfilter scales while the flux of energy in subfilter scales is reduced by the limit on local small-scale to small-scale interactions $[14,45]$.

The LANS- $\alpha$ model is given by,

$$
\begin{aligned}
\partial_{t} \mathbf{v}+\omega \times \overline{\mathbf{v}} & =-\nabla \pi+v \nabla^{2} \mathbf{v}, \\
\nabla \cdot \mathbf{v} & =\nabla \cdot \overline{\mathbf{v}}=0 .
\end{aligned}
$$

From the identity, $\omega \times \overline{\mathbf{v}}=\overline{\mathbf{v}} \cdot \nabla \mathbf{v}+(\nabla \overline{\mathbf{v}})^{T} \cdot \mathbf{v}-\nabla(\overline{\mathbf{v}} \cdot \mathbf{v})$, we see that it differs from NavierStokes both in advection by the smoothed velocity and the addition of a second nonlinear term associated with the conservation of circulation. Traditionally, LANS- $\alpha$ is used with an inverse Helmholtz operator as the filter: $\bar{v}_{i}=\left(1-\alpha^{2} \partial_{j j}\right)^{-1} v_{i}$. In this case, LANS- $\alpha$ can be written as a LES, (1), with

$$
\bar{\tau}_{i j}^{\alpha}=\left(1-\alpha^{2} \partial_{j j}\right)^{-1} \alpha^{2}\left(\partial_{m} \bar{v}_{i} \partial_{m} \bar{v}_{j}+\partial_{m} \bar{v}_{i} \partial_{j} \bar{v}_{m}-\partial_{i} \bar{v}_{m} \partial_{j} \bar{v}_{m}\right)
$$

The model allows for a reduction in resolution without changing (or supplementing) the dissipative term by instead altering the SFS scaling properties. Near wavenumber, $k=2 \pi / \alpha$, the $H_{\alpha}^{1}$ energy spectrum is predicted to transition from $k^{\beta}$ with $\beta=-5 / 3$ at larger scales to $\beta=-1$ at smaller scales [14]. Consequently, dissipation $\left(\Omega_{(\alpha)}(k)=k^{2} E_{(\alpha)}(k)\right)$ goes as $k^{1}$ instead of $k^{1 / 3}$ and the same amount of power is dissipated in fewer scales. The change in spectral scaling also predicts a resolution requirement in degrees of freedom, dof, for LANS [14],

$$
d o f_{\alpha} \sim \alpha^{-1} \operatorname{Re}^{3 / 2}
$$


Fig. 1 Energy spectrum for LANS- $\alpha$ (solid line) with filter scale $\alpha=2 \pi / 3$ (vertical dashed line). The SFS power law is well approximated by $k^{+1}$. Insert shows thresholded cubed velocity increment $\left|\delta \bar{v}_{\|}(2 \pi / 10)\right|^{3}<10^{-2}$ in black. These regions do not contribute to the turbulent cascade of energy to smaller scales and are identified with rigid bodies. A spectrum of only the white regions (dashed line) is consistent with the predicted $k^{-1}$ scaling outside rigid bodies

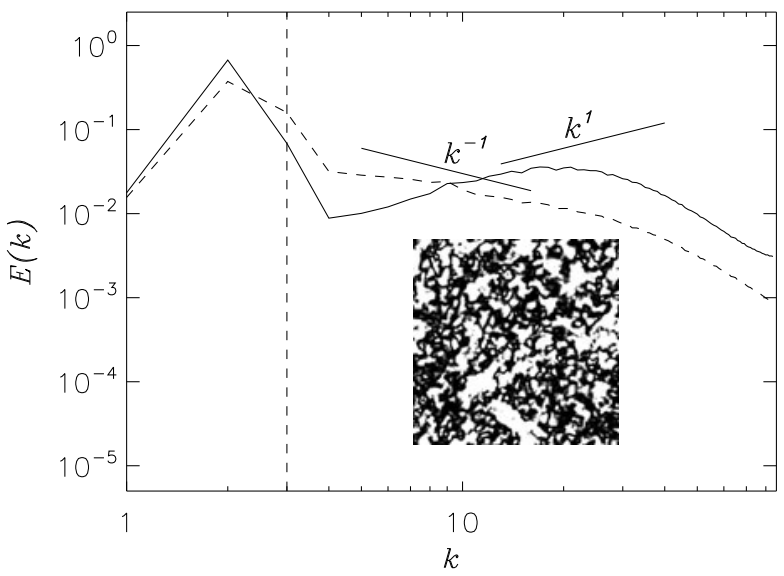

which has been confirmed in numerical experiments [45]. Once $d o f_{\alpha}$ has been resolved, further resolution yields no change in the numerical solution: LANS- $\alpha$ is a grid-independent SFS model. When compared with the dof for Navier-Stokes,

$$
d o f_{N S} \sim R e^{9 / 4}
$$

we see that LANS- $\alpha$ should improve as a SFS model for larger $R e$. This was an encouraging prediction as LANS- $\alpha$ compared well with dynamic eddy viscosity [42] and dynamic mixed (similarity) eddy viscosity [18] at moderate $R e$.

We have, however, found that LANS- $\alpha$ develops a problem at large $R e$ : it develops a positive-exponent power-law bump in its small-scale energy spectrum and a contamination of superfilter-scale spectral properties [45]. To investigate the SFS physics responsible for this, we employ (as the antithesis of a LES) a filter $1 / 3$ the size of our $256^{3}$ computational cube in a pseudo-spectral calculation [20, 21] with a Taylor-Green (TG) forcing [51] and $R e \approx 8000$. As shown in Fig. 1, the observed scaling law is $k^{+1}$. This was shown to be associated with the formation in the flow of passively swept regions, called rigid bodies [45]. These form as a consequence of disallowing sub- $\alpha$-scale fluctuations to interact with each other in the closure approximation. A rigid body cannot support longitudinal velocity increments: $\delta \bar{v}_{\|}(l) \equiv[\overline{\mathbf{v}}(\mathbf{x})-\overline{\mathbf{v}}(\mathbf{x}+\mathbf{l})] \cdot \mathbf{l} / l=0$. This dimensionally predicts a scaling relation, $\delta \bar{v} \sim l^{0}$, and, with $v \sim\left(1+\alpha^{2} k^{2}\right) \bar{v} \sim \alpha^{2} k^{2} \bar{v}$ for $l \ll \alpha$, an energy spectrum of

$$
E_{\alpha}(k) \sim \bar{v} v k^{-1} \sim k^{1}
$$

which is compatible with the observed SFS energy spectrum. Inside rigid bodies there can be no turbulent cascade of energy to smaller scales (since there are no internal degrees of freedom). From the Kármán-Howarth theorem, we should then expect to be able to detect rigid bodies by visualizing the cubed velocity increments (which are proportional to the energy flux). The regions which correspond to negligible flux are shown as black in the inset of Fig. 1. Filtering these regions out, allows us to obtain a (convolved) energy spectra for the remaining white portion of the flow. This spectrum is shown as a dashed line in Fig. 1 and has a negative spectral slope close to the predicted $k^{-1}$ spectrum. The resulting picture of the model's behavior is to produce two spatially separate scalings. The white portions of the flow possess the predicted LANS- $\alpha$ scaling and are responsible for the observation of the 
predicted $d o f_{\alpha}$. The black portions are rigid bodies whose $k^{1}$ energy spectrum dominates over $k^{-1}$ for large $k$ and are responsible for the observed spectral contamination.

LANS- $\alpha$ stops the turbulent cascade by the physical sweeping of spatial scales smaller than $\alpha$ by flows at spatial scales larger than $\alpha$. The motion of the subfilter scales is seen as rigid rotation plus translation, tumbling along helical paths generated by the flows of the larger scales (instead of, for example, making fluid blobs that are smaller than $\alpha$ all share the same time-scale, leading to a $k^{-3}$ spectrum like in 2D turbulence). Because of the $k^{+1}$ spectrum associated with rigid bodies, however, LANS- $\alpha$ may not considered useful as a SFS model. Therefore, there is no need for further tests of LANS- $\alpha$ against newer models such as the Variational Multiscale Method [26]. Note, however, that suitable spectra can be obtained with a modified viscous length scale [29].

\subsection{The Influence of Circulation on Rigid Bodies}

The formation of rigid bodies in LANS- $\alpha$ limits the reduction of numerical dof saved compared to Navier-Stokes to a factor of $1 / 12$ regardless of $R e$ [45]. It is desirable, then, to alter the model in such a way to prevent the formation of rigid bodies. Truncation of the SFS stress tensor, (4), to the first term results in the Clark- $\alpha$ model [8],

$$
\begin{aligned}
\partial_{t} \mathbf{v}+\left(1-\frac{1}{2} \alpha^{2} \nabla^{2}\right)(\overline{\boldsymbol{\omega}} \times \overline{\mathbf{v}})-\frac{1}{2} \alpha^{2}\left[\left(\nabla^{2} \overline{\boldsymbol{\omega}}\right) \times \overline{\mathbf{v}}+\overline{\boldsymbol{\omega}} \times\left(\nabla^{2} \overline{\mathbf{v}}\right)\right] & =-\nabla \mathcal{P}+v \nabla^{2} \mathbf{v}, \\
\nabla \cdot \mathbf{v} & =\nabla \cdot \overline{\mathbf{v}}=0,
\end{aligned}
$$

and to the first two terms results in the Leray- $\alpha$ model $[17,18]$,

$$
\begin{gathered}
\partial_{t} \mathbf{v}+\overline{\mathbf{v}} \cdot \nabla \mathbf{v}=-\nabla p+v \nabla^{2} \mathbf{v}, \\
\nabla \cdot \mathbf{v}=\nabla \cdot \overline{\mathbf{v}}=0 .
\end{gathered}
$$

Both these models are regularizations and conserve the total energy of the flow $[8,17,18]$. They do not, however, conserve the helicity nor the small-scale circulation. Considering the rotational properties of a rigid body (in the absence of viscous friction), these models' circulation properties may be incompatible with rigid body formation. Indeed, while LANS$\alpha$ exhibits a positive-exponent power law in this case $(\alpha=2 \pi / 13, \operatorname{Re} \approx 3300$, TG forcing), both Clark- $\alpha$ and Leray- $\alpha$ are free from this signature of rigid body formation (Fig. 2a). However, LANS- $\alpha$ 's intermittency properties are more similar to Navier-Stokes than the other two models (Fig. 2b).

\subsection{Computability and Interpretation of $H_{\alpha}^{1}$ Norm Regularizations}

It has been reported recently that Clark- $\alpha$ and LANS- $\alpha$ have poor SFS model performance and, in fact, have instabilities in simulations with periodic boundary conditions [9]. This result highlights the importance of correctly interpreting $H_{\alpha}^{1}$ norm regularizations. To illustrate this, we compute $256^{3}$ DNS and $64^{3}$ models runs for case $3 a$ of [9] decaying Taylor-Green vortex with $v=2.5 \cdot 10^{-3}$ (time step, $d t=3 \cdot 10^{-3}$ for all simulations). In Figs. 3 and 4 , we display results for the models employing a filter width $\alpha=2 \pi / 16$ (results with $\alpha=2 \pi / 24$ are closer to that of the DNS). Figure 3 a shows the time evolution of the total kinetic energy in the super-filter scales,

$$
t k e \equiv \int_{0}^{k_{\alpha}} E_{(\alpha)}(k),
$$


Fig. 2 (Color online)

(a) Compensated energy spectra ( $2 \pi / \alpha$, vertical dashed line) for Navier-Stokes (solid black), LANS- $\alpha$ (blue dash-dotted), Clark- $\alpha$ (green dashed), and Leray- $\alpha$ (red dotted).

(b) Normalized structure function scaling exponent $\xi_{p} / \xi_{3}$ versus order $p$. Clark- $\alpha$ is the best approximation for the superfilter-scale spectrum, whereas high-order intermittency properties are best reproduced by LANS- $\alpha$ [47]
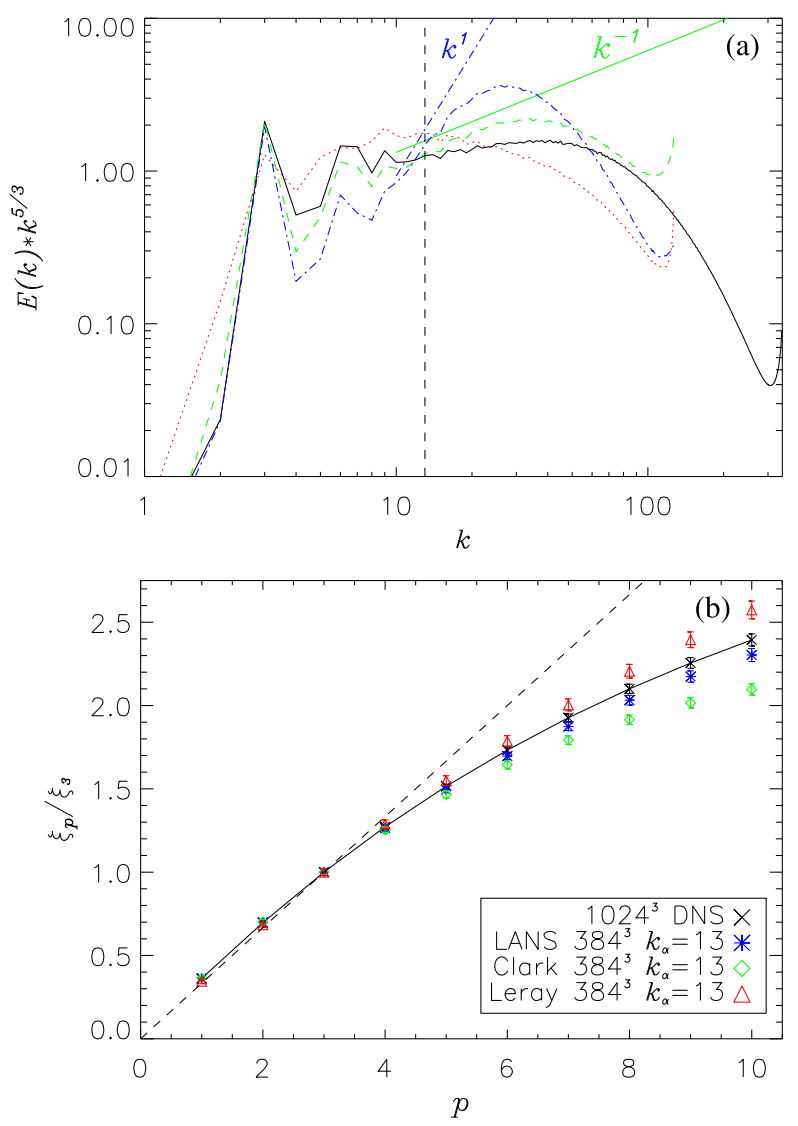

where $k_{\alpha}=2 \pi / \alpha$. Incorrectly applying the $L^{2}$ norm, $E=|\mathbf{v}|^{2} / 2$, to Clark- $\alpha$ and LANS- $\alpha$ leads to the interpretation that they deviate significantly from the DNS due to slower energy decay. Correctly applying the $H_{\alpha}^{1}$ norm, $E_{\alpha}=\overline{\mathbf{v}} \cdot \mathbf{v} / 2$, shows that the models are, in fact, doing quite well as can be seen in the plot of the dissipation rate of energy, $\varepsilon$ (Fig. $3 \mathrm{~b}$ ). The qualitative properties of Lagrangian models is that they have the same invariants as the primitive equations, albeit in a different norm. Since it is these invariants which may very well influence the dynamics, as for example shown in [12] where Kolmogorov spectra are present at intermediate times at large scale in ideal (Euler) three-dimensional fluid flows, one must compare the behavior of one set of invariants to the other set in the Lagrangian modeling formulation. Similarly, the $L^{2}$ norm spectra for Clark- $\alpha$ and LANS- $\alpha$ in Fig. 4a appear under-dissipative at high wave numbers, while the correct $H_{\alpha}^{1}$ norm spectra are closer to the DNS. We also conduct Clark- $\alpha$ and LANS- $\alpha$ computations for case $3 b$ of [9]: $v=1 / 3000$, $\alpha=2 \pi / 32$ and $384^{3}$ grid points (Fig. $4 \mathrm{~b}$ ). We find no signs of instability. The particular numerical expression of the models we used is given in (3) and (8). These differ from (8) and (9) in [9] and it is known that discretized pseudospectral operators depend on their algorithmic form (e.g., $\left.\nabla \psi^{2} \neq 2 \psi \nabla \psi[7,13]\right)$. This is likely the source of their observed instability; however a recently discovered deficiency in high-order low-storage Runge-Kutta schemes [6] may also impact numerical implementation of the models. For this reason, our calculations are made with second-order Runge-Kutta in time. It is worth pointing out that Clark- $\alpha$ is nearly identical to the Rational Large Eddy Simulation (RLES) model [15] which has 
Fig. 3 (Color online) Case 3a, $R e \approx 400$. Line styles are as in Fig. 2a for correct norms with the addition of $L^{2}$ norm energy for Clark- $\alpha$ (pink dash-triple-dotted) and for LANS- $\alpha$ (cyan long-dashed). (a) Filtered kinetic energy, tke. (b) Dissipation, $\varepsilon=d E / d t$, versus time, $t$
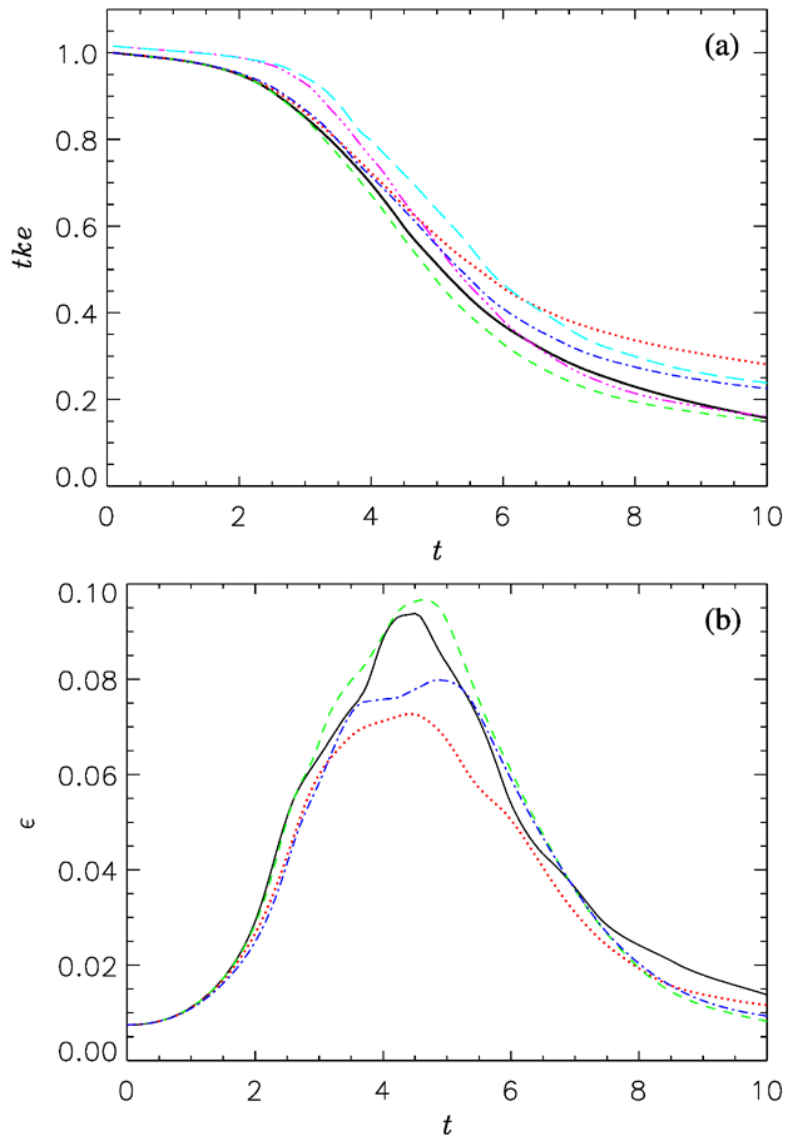

been shown to be unstable for non-periodic boundaries [5, 28]. Both our work and that of [9] have employed periodic boundary conditions only, but extra precautions must be taken for stability in the non-periodic case [5, 28].

\section{MHD: Circulation and Outlook for LES}

In MHD, the circulation properties are quite different since small-scale circulation is broken by the Lorentz force $\mathbf{j} \times \mathbf{b}$, with $\mathbf{j}=\boldsymbol{\nabla} \times \mathbf{b}$ the current, $\mathbf{b}$ being the induction. This force acts as source (sink) of circulation, $\Gamma$, as opposed to the insufficient modeling of $\Gamma$ in the Leray- $\alpha$ and Clark- $\alpha$ models. This can be seen in Kelvin's theorem,

$$
\frac{d}{d t} \Gamma=\frac{d}{d t} \oint_{\mathcal{C}} \mathbf{v} \cdot d \mathbf{r}=\oint_{\mathcal{C}} \mathbf{j} \times \mathbf{b} \cdot d \mathbf{r}
$$

This may prevent the formation of rigid bodies even while conserving all the correct physical properties of the flow. 
Fig. 4 (Color online)

(a) Case $3 \mathrm{a}, \operatorname{Re} \approx 400$ : energy spectra after peak of dissipation, $t \in[4.5,5.5]$. Line styles are as in Fig. 3. Vertical dashed line is $2 \pi / \alpha$. (b) Case $3 b, R e \approx 3000:$ dissipation, $\varepsilon$, versus time, $t$ for Clark- $\alpha$ (light green) and for LANS- $\alpha$ (dark blue)
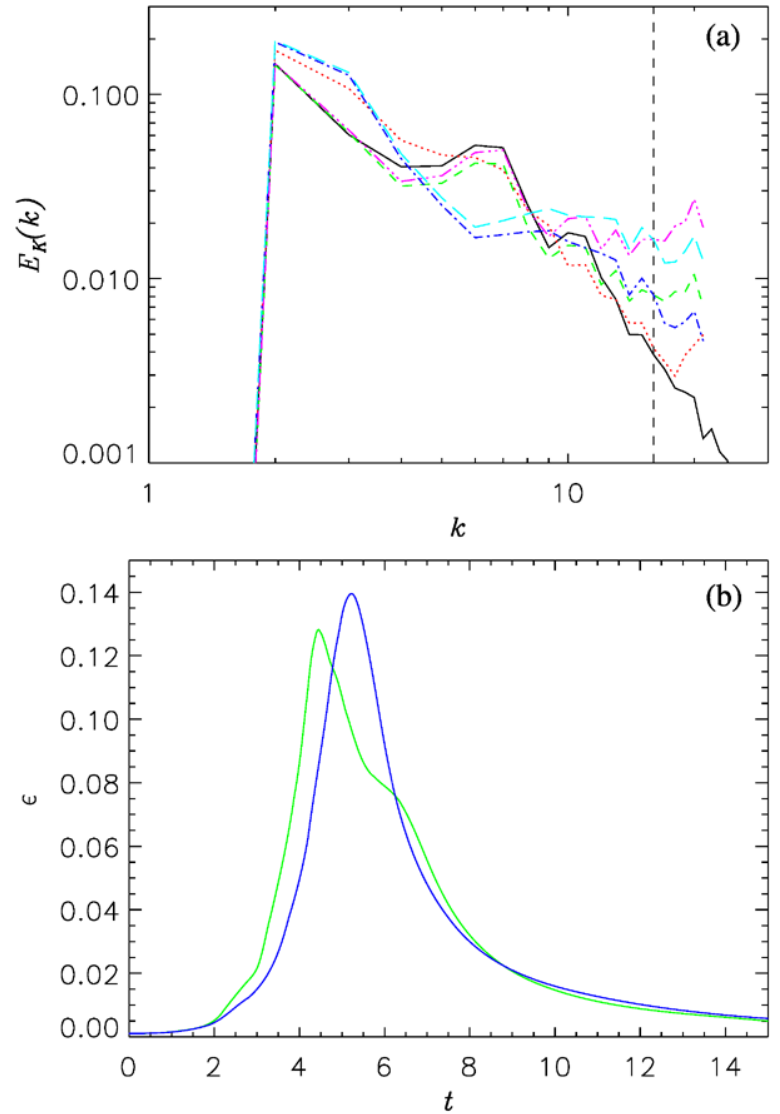

The LES equations for MHD are given by

$$
\begin{aligned}
\partial_{t} \overline{\mathbf{v}}+\overline{\boldsymbol{\omega}} \times \overline{\mathbf{v}} & =\overline{\mathbf{j}} \times \overline{\mathbf{b}}-\nabla \bar{\Pi}+v \nabla^{2} \overline{\mathbf{v}}-\nabla \cdot \bar{\tau}, \\
\partial_{t} \overline{\mathbf{b}} & =\nabla \times(\overline{\mathbf{v}} \times \overline{\mathbf{b}})+\eta \nabla^{2} \overline{\mathbf{b}}-\nabla \cdot \bar{\tau}^{b},
\end{aligned}
$$

where $\eta$ is the magnetic diffusivity, $\Pi=P+|\mathbf{b}|^{2} / 2$ the modified pressure, $\bar{\tau}$ is the Reynolds SFS stress tensor,

$$
\bar{\tau}_{i j}=\overline{v_{i} v_{j}}-\bar{v}_{i} \bar{v}_{j}-\left(\overline{b_{i} b_{j}}-\bar{b}_{i} \bar{b}_{j}\right)
$$

and $\bar{\tau}^{b}$ is the electromotive-force SFS stress tensor,

$$
\bar{\tau}_{i j}^{b}=\overline{b_{i} v_{j}}-\bar{b}_{i} \bar{v}_{j}-\left(\overline{v_{i} b_{j}}-\bar{v}_{i} \bar{b}_{j}\right)
$$

Note that the extension of eddy viscosity to eddy resistivity employs the usual form for $\bar{\tau}$ involving only the filtered velocity while the expression for $\bar{\tau}^{b}$ similarly only involves the filtered magnetic field [52]. Meanwhile, (13) and (14) make it explicitly clear that interactions between the two fields at subfilter scales must be taken into account. 
Another problem with extending eddy-viscosity concepts to MHD is that they can be related to a known power law of the energy spectrum [11]. This is inappropriate for MHD as neither kinetic nor magnetic energy is a conserved quantity and the general expression of the energy spectrum is not known at this time [19, 27, 31, 35, 37]. Additionally, MHD has been shown to have nonlocal interactions between large and small (superfilter and subfilter) scales [1] (e.g., in the Batchelor viscous-inductive regime, $v \gg \eta$, where energy is transferred directly from viscous-scale, $l_{v}$, eddies to small-scale magnetic field, $l \ll l_{v}[3]$ ). This complex interaction is a challenge in general for MHD-LES, but may be an advantage for the Lagrangian-averaged approach as energy exchanges with sub- $\alpha$ scales may disrupt rigid body formation. Some limited case MHD LES include the cross-helicity model [44] assuming alignment between the fields and the low magnetic Re LES [30, 49]. Extensions of spectral models to MHD based on two-point closure formulations of the dynamical equations proposed recently look promising in the analysis of turbulent flows and of the dynamo mechanism [4]. Approximate deconvolution models for MHD [32] are theoretically appealing, but have yet to be tested on non-laminar flows. However, there are many regimes of MHD dependent on the ratios between the various conserved quantities and $v / \eta$ : there has yet to be demonstrated a generally applicable LES for MHD.

Fig. 5 Kinetic (a) and magnetic (b) energy spectra $(2 \pi / \alpha$ vertical dashed line). The largest scales are affected by differences from the MHD DNS (solid lines) in initial conditions. LAMHD- $\alpha$ (dashed lines) exhibits neither the positive power-law nor the superfilter-scale spectral contamination associated with high $\operatorname{Re}$ LANS- $\alpha$
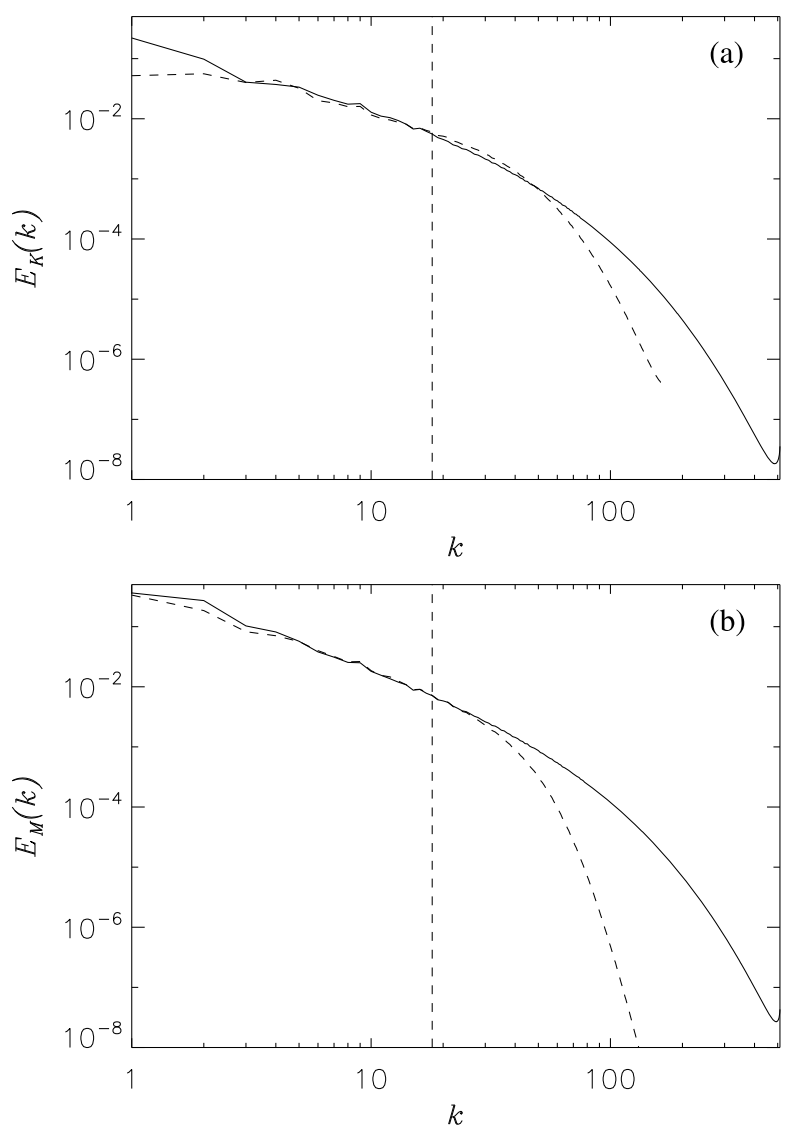


\subsection{LAMHD- $\alpha$ and Absence of Rigid Bodies}

The Lagrangian-averaged MHD $\alpha$-model (LAMHD- $\alpha$ ) [23, 24, 43] is given by, where the velocity if filtered as before and $\overline{\mathbf{b}}=\left(1-\alpha_{M}^{2} \nabla^{2}\right)^{-1} \mathbf{b}$ :

$$
\begin{aligned}
\partial_{t} \mathbf{v}+\omega \times \overline{\mathbf{v}} & =\mathbf{j} \times \overline{\mathbf{b}}-\nabla \pi+v \nabla^{2} \mathbf{v}, \\
\partial_{t} \overline{\mathbf{b}} & =\nabla \times(\overline{\mathbf{v}} \times \overline{\mathbf{b}})+\eta \nabla^{2} \mathbf{b}, \\
\nabla \cdot \mathbf{v} & =\nabla \cdot \overline{\mathbf{v}}=\nabla \cdot \mathbf{b}=\nabla \cdot \overline{\mathbf{b}}=0 .
\end{aligned}
$$

LAMHD- $\alpha$ may be written as a MHD-LES, (12), for the case $\alpha_{M}=\alpha$ [46, 48], which we study here. The model preserves the ideal quadratic invariants of MHD (in the $H_{\alpha}^{1}$ norm) as well as Alfvén's theorem for frozen-in field lines [24]. Physically, it supports Alfvén waves at all scales while slowing and hyper-diffusively damping waves with wavelengths, $\lambda$, smaller than $\alpha$ [48]. In examinations of its SFS physical properties LAMHD- $\alpha$ (dashed lines) displays neither positive-exponent power-law scaling nor superfilter-scale spectral contamination (see Fig. 5). Under similar conditions LANS- $\alpha$ (not shown) displays these signs of rigid body formation. Further examinations with larger filters and higher $R e$ were unable to unravel any sign that rigid bodies form for LAMHD- $\alpha$ [48].

Fig. 6 (Color online) Time evolution of total (solid), magnetic (dashed), and kinetic (dotted) energies (a) and total dissipation, $\varepsilon$, (b) for $1024^{3}$ DNS (black/solid), $168^{3}$ LAMHD- $\alpha$ (blue/dash-dotted), and $168^{3}$ no-model (red/dotted). LAMHD- $\alpha$ provides no improvement in prediction of global quantities over an under-resolved DNS
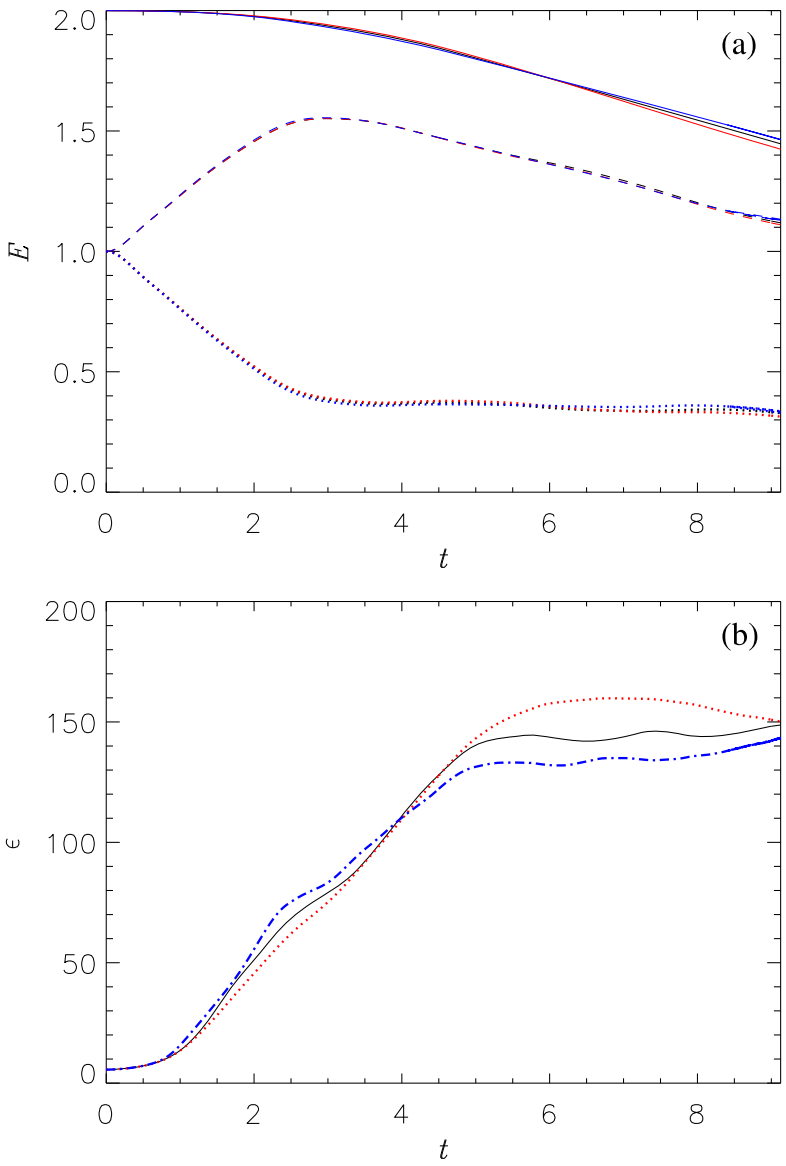


\subsection{LAMHD- $\alpha$ as a SFS Model}

Since LAMHD- $\alpha$ did not display any signs of rigid body spectral contamination, we test it as a SFS model for large kinetic and magnetic Reynolds numbers, $\approx 3300$. A DNS of MHD is computed at a resolution of $1024^{3}$. The initial conditions for $\mathbf{v}$ and $\mathbf{b}$ are a superposition of $\mathrm{ABC}$ modes [16] with random phases and wavenumbers $k \in[1,4]$. No external forcing is applied and the total energy is allowed to freely decay. LAMHD- $\alpha$ is computed for identical conditions at a resolution of $N^{3}=168^{3}$ with the same $R e$ and a filter size $\alpha=6 \Delta=2 \pi / 28$. As a base-level comparison we also compute an under-resolved (or no-model) solution of the MHD equations at $N^{3}=168^{3}$. Time evolution of the total energies and enstrophy are shown in Fig. 6. In comparison with under-resolving MHD, LAMHD- $\alpha$ shows errors of approximately the same magnitude in these global quantities. Comparisons of energy spectra (Fig. 7), however, show an improvement in predictive quality for LAMHD- $\alpha$, especially for the magnetic energy spectrum. As turbulence develops, energy begins to pile up at smallscales and deplete at intermediate scales for $168^{3}$ MHD. LAMHD- $\alpha$ improves the prediction of superfilter-scale spectra compared to no SFS model.

Cross-sections of $|\mathbf{j}|^{2}$, shown in Fig. 8 at $t=8.4$ indicate that LAMHD- $\alpha$ finds sharper and better defined, more intermittent current sheets than the under-resolved run compared to the DNS.

Fig. 7 Compensated kinetic (a) and magnetic (b) energy spectra averaged over $t \in[8,8.8]$. Line styles are as in Fig. 6. $(2 \pi / \alpha$ vertical dashed line). Energy piles up at small scales in the no-model approach (under-resolved DNS) and LAMHD- $\alpha$ is seen to provide improved predictions of the superfilter-scale spectra, especially for the magnetic field
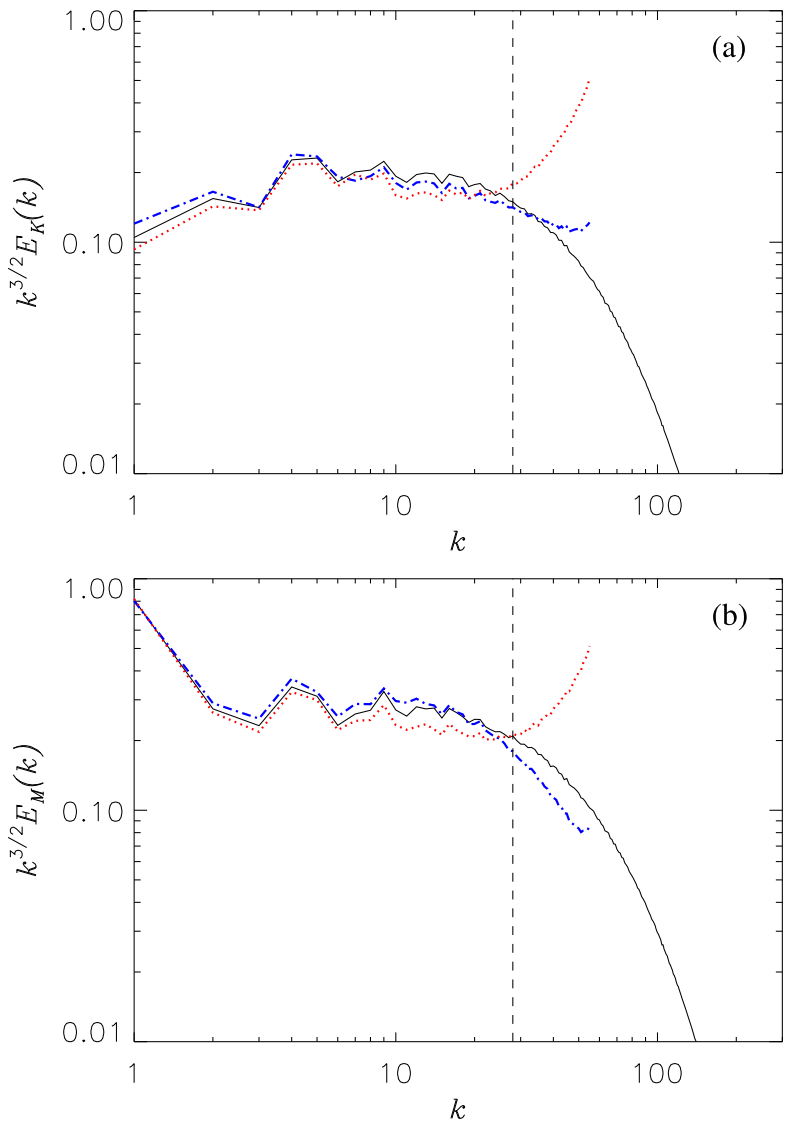


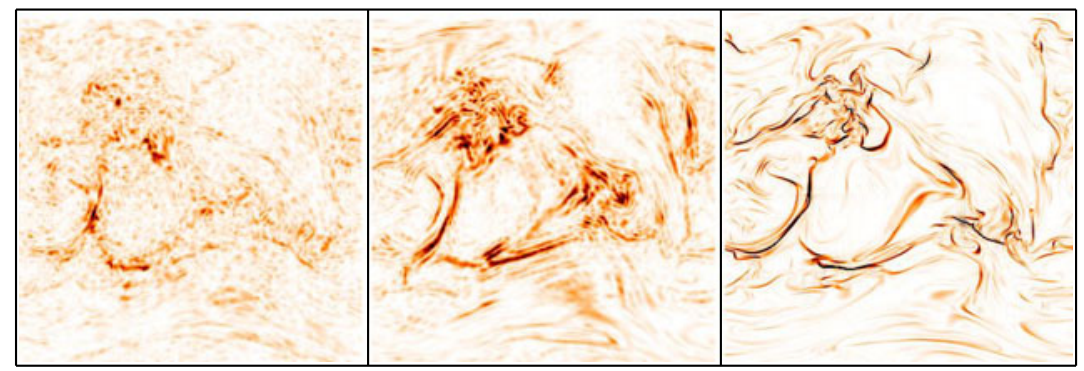

Fig. 8 Cross sections of square current, $j^{2}$, at $t=8.4$ for no-model $168^{3}$ (left), $168^{3}$ LAMHD- $\alpha$ (center), and $1024^{3}$ DNS (right) at full resolution. LAMHD- $\alpha$ provides a much better capturing of the intermittent current sheets than the under-resolved solution

\section{Summary}

Incompressible LANS- $\alpha$, while it performed well at moderate Reynolds number is limited as a high $R e$ SFS model. Due to its strong suppression of spectrally local interactions at subfilter-scales, and consistent with its conservation of small-scale circulation, LANS- $\alpha$ develops rigid bodies which contaminate the superfilter-scale energy spectrum. In contrast, Clark- $\alpha$ and Leray- $\alpha$, neither of which conserve small-scale circulation do not develop energy-spectrum contamination from rigid bodies. LANS- $\alpha$, however, best matches the intermittency properties of Navier-Stokes fluid turbulence.

In MHD, a mechanism for local small-scale transfer is the interaction of small-scale Alfvén waves. As LAMHD- $\alpha$ supports Alfvén waves at all scales while slowing and hyperdiffusively damping those with wavelength $\lambda<\alpha$, it more gently suppresses SFS local interactions than LANS- $\alpha$. This together with the greater nonlocality in MHD and the Lorentz-force source of small-scale circulation, inhibits the formation of rigid bodies in LAMHD- $\alpha$. It appears to retain the good intermittency properties of LANS- $\alpha$ without its poor spectral properties. For this reason, we find LAMHD- $\alpha$ to be a viable model at high $R e$ in 3D. As LAMHD- $\alpha$ has been previously found to reproduce the difficult to model properties of MHD at high $R e$ in 2D [41] and moderate $R e$ in 3D [40], we believe it will prove to be a generally applicable MHD LES, in many instances in geophysics and astrophysics where magnetic fields are known to be important dynamically.

\section{References}

1. Alexakis, A., Mininni, P.D., Pouquet, A.: Shell-to-shell energy transfer in magnetohydrodynamics. I. Steady state turbulence. Phys. Rev. E 72(4), 046301 (2005)

2. Aluie, H., Eyink, G.L.: Localness of energy cascade in hydrodynamic turbulence. II. Sharp spectral filter. Phys. Fluids 21(11), 115108 (2009)

3. Aluie, H., Eyink, G.L.: Scale-locality of magnetohydrodynamic turbulence. ArXiv e-prints (2009)

4. Baerenzung, J., Politano, H., Ponty, Y., Pouquet, A.: Spectral modeling of magnetohydrodynamic turbulent flows. Phys. Rev. E 78(2), 026310 (2008)

5. Berselli, L.C., Grisanti, C.R.: On the consistency of the rational large eddy simulation model. Comput. Vis. Sci. 6(2-3), 75-82 (2004)

6. Brachet, M.E., Mininni, P.D., Rosenberg, D.L., Pouquet, A.: High-order low-storage explicit RungeKutta schemes for equations with quadratic nonlinearities. ArXiv e-prints (2008)

7. Canuto, C., Yousuff Hussaini, M., Quarteroni, A., Zang, T.A.: Spectral Methods in Fluid Dynamics. Springer, New York (1988) 
8. Cao, C., Holm, D.D., Titi, E.S.: On the Clark $\alpha$ model of turbulence: global regularity and long-time dynamics. J. Turbul. 6, N20 (2005)

9. Chandy, A., Frankel, S.: Regularization-based sub-grid scale (SGS) models for large eddy simulations (LES) of high-Re decaying isotropic turbulence. J. Turbul. 10, 25 (2009)

10. Chen, S., Foias, C., Holm, D.D., Olson, E., Titi, E.S., Wynne, S.: Camassa-Holm equations as a closure model for turbulent channel and pipe flow. Phys. Rev. Lett. 81, 5338-5341 (1998)

11. Chollet, J.-P., Lesieur, M.: Parameterization of small scales of three-dimensional isotropic turbulence utilizing spectral closures. J. Atmos. Sci. 38, 2747-2757 (1981)

12. Cichowlas, C., Bonaïti, P., Debbasch, F., Brachet, M.: Effective dissipation and turbulence in spectrally truncated Euler flows. Phys. Rev. Lett. 95(26), 264502 (2005)

13. Dahlburg, J.P., Montgomery, D., Doolen, G.D., Matthaeus, W.H.: Large-scale disruptions in a currentcarrying magnetofluid. J. Plasma Phys. 35, 1-42 (1986)

14. Foias, C., Holm, D.D., Titi, E.S.: The Navier-Stokes-alpha model of fluid turbulence. Physica D, Nonlinear Phenom. 152-153, 505-519 (2001)

15. Galdi, G.P., Layton, W.J.: Approximation of the larger eddies in fluid motions. II: A model for spacefiltered flow. Math. Models Methods Appl. Sci. 10, 343-350 (2000)

16. Galloway, D., Frisch, U.: Dynamo action in a family of flows with chaotic streamlines. Geophys. Astrophys. Fluid Dyn. 36, 53-83 (1986)

17. Geurts, B.J., Holm, D.D.: Regularization modeling for large-eddy simulation. Phys. Fluids 15, L13-L16 (2003)

18. Geurts, B.J., Holm, D.D.: Leray and LANS- $\alpha$ modelling of turbulent mixing. J. Turbul. 7(10), 1-33 (2006)

19. Goldreich, P., Sridhar, S.: Toward a theory of interstellar turbulence. 2: Strong Alfvénic turbulence. Astrophys. J. 438, 763-775 (1995)

20. Gómez, D.O., Mininni, P.D., Dmitruk, P.: MHD simulations and astrophysical applications. Adv. Space Res. 35, 899-907 (2005)

21. Gómez, D.O., Mininni, P.D., Dmitruk, P.: Parallel simulations in turbulent MHD. Phys. Scr. T 116, 123127 (2005)

22. Guermond, J.-L.: On the use of the notion of suitable weak solutions in CFD. Int. J. Numer. Methods Fluids 57, 1153-1170 (2008)

23. Holm, D.D.: Averaged Lagrangians and the mean effects of fluctuations in ideal fluid dynamics. Physica D, Nonlinear Phenom. 170, 253-286 (2002)

24. Holm, D.D.: Lagrangian averages, averaged Lagrangians, and the mean effects of fluctuations in fluid dynamics. Chaos 12, 518-530 (2002)

25. Holm, D.D., Marsden, J.E., Ratiu, T.S.: The Euler-Poincaré equations and semidirect products with applications to continuum theories. Adv. Math. 137, 1-81 (1998)

26. Hughes, T.J.R., Mazzei, L., Oberai, A.A., Wray, A.A.: The multiscale formulation of large eddy simulation: Decay of homogeneous isotropic turbulence. Phys. Fluids 13, 505-512 (2001)

27. Iroshnikov, P.S.: Turbulence of a conducting fluid in a strong magnetic field. Soviet Astron. 7, 566 (1964)

28. John, V.: An assessment of two models for the subgrid scale tensor in the rational LES model. J. Comput. Appl. Math. 173(1), 57-80 (2005)

29. Kim, T.-Y., Cassiani, M., Albertson, J.D., Dolbow, J.E., Fried, E., Gurtin, M.E.: Impact of the inherent separation of scales in the Navier-Stokes- $\alpha \beta$ equations. Phys. Rev. E 79(4), 045307 (2009)

30. Knaepen, B., Moin, P.: Large-eddy simulation of conductive flows at low magnetic Reynolds number. Phys. Fluids 16, 1255 (2004)

31. Kraichnan, R.H.: Inertial-range spectrum of hydromagnetic turbulence. Phys. Fluids 8, 1385-1387 (1965)

32. Labovschii, A., Trenchea, C.: Approximate deconvolution models for magnetohydrodynamics. Technical report, University of Pittsburgh (2010)

33. Larios, A., Titi, E.S.: On the higher-order global regularity of the inviscid Voigt-regularization of threedimensional hydrodynamic models. ArXiv e-prints (2009)

34. Lax, P.D., Richtmyer, R.D.: Survey of the stability of linear finite difference equations. Commun. Pure Appl. Math. 9, 267-293 (1956)

35. Lee, E., Brachet, M.E., Pouquet, A., Mininni, P.D., Rosenberg, D.: Lack of universality in decaying magnetohydrodynamic turbulence. Phys. Rev. E 81(1), 016318 (2010)

36. Levant, B., Ramos, F., Titi, E.S.: On the statistical properties of the 3D incompressible Navier-StokesVoigt model. ArXiv e-prints (2009)

37. Mason, J., Cattaneo, F., Boldyrev, S.: Numerical measurements of the spectrum in magnetohydrodynamic turbulence. Phys. Rev. E 77(3), 036403 (2008)

38. Meneveau, C., Katz, J.: Scale-invariance and turbulence models for large-eddy simulation. Annu. Rev. Fluid Mech. 32, 1-32 (2000) 
39. Mininni, P.D., Alexakis, A., Pouquet, A.: Nonlocal interactions in hydrodynamic turbulence at high Reynolds numbers: The slow emergence of scaling laws. Phys. Rev. E 77(3), 036306 (2008)

40. Mininni, P.D., Montgomery, D.C., Pouquet, A.: Numerical solutions of the three-dimensional magnetohydrodynamic $\alpha$ model. Phys. Rev. E 71(4), 046304 (2005)

41. Mininni, P.D., Montgomery, D.C., Pouquet, A.G.: A numerical study of the alpha model for twodimensional magnetohydrodynamic turbulent flows. Phys. Fluids 17(3), 035112 (2005)

42. Mohseni, K., Kosović, B., Shkoller, S., Marsden, J.E.: Numerical simulations of the Lagrangian averaged Navier-Stokes equations for homogeneous isotropic turbulence. Phys. Fluids 15, 524-544 (2003)

43. Montgomery, D.C., Pouquet, A.: An alternative interpretation for the Holm "alpha model". Phys. Fluids 14(9), 3365-3366 (2002)

44. Müller, W.-C., Carati, D.: Dynamic gradient-diffusion subgrid models for incompressible magnetohydrodynamic turbulence. Phys. Plasmas 9, 824-834 (2002)

45. Pietarila Graham, J., Holm, D., Mininni, P., Pouquet, A.: Highly turbulent solutions of the Lagrangianaveraged Navier-Stokes alpha model and their large-eddy-simulation potential. Phys. Rev. E 76, 056310 (2007)

46. Pietarila Graham, J., Holm, D.D., Mininni, P., Pouquet, A.: Inertial range scaling, Kármán-Howarth theorem, and intermittency for forced and decaying Lagrangian averaged magnetohydrodynamic equations in two dimensions. Phys. Fluids 18, 045106 (2006)

47. Pietarila Graham, J., Holm, D.D., Mininni, P.D., Pouquet, A.: Three regularization models of the NavierStokes equations. Phys. Fluids 20(3), 035107 (2008)

48. Pietarila Graham, J., Mininni, P.D., Pouquet, A.: Lagrangian-averaged model for magnetohydrodynamic turbulence and the absence of bottlenecks. Phys. Rev. E 80(1), 016313 (2009)

49. Ponty, Y., Politano, H., Pinton, J.-F.: Simulation of induction at low magnetic Prandtl number. Phys. Rev. Lett. 92(14), 144503 (2004)

50. Ramos, F., Titi, E.S.: Invariant measures for the 3D Navier-Stokes-Voigt equations and their NavierStokes limit. ArXiv e-prints (2009)

51. Taylor, G.I., Green, A.E.: Mechanism of the production of small eddies from large ones. Proc. R. Soc. Lond. A 158, 499 (1937)

52. Theobald, M.L., Fox, P.A., Sofia, S.: A subgrid-scale resistivity for magnetohydrodynamics. Phys. Plasmas 1, 3016-3032 (1994)

53. van Reeuwijk, M., Jonker, H.J.J., Hanjalić, K.: Leray- $\alpha$ simulations of wall-bounded turbulent flows. Int. J. Heat Fluid Flow 30, 1044 (2009) 